\title{
SIMPLY TRANSITIVE GROUPS AND KÄHLER STRUCTURES ON HOMOGENEOUS SIEGEL DOMAINS
}

\author{
BY
}

JOSEF DORFMEISTER

\begin{abstract}
We determine the Lie algebras of all simply transitive groups of automorphisms of a homogeneous Siegel domain $D$ as modifications of standard normal $j$-algebras. We show that the Lie algebra of all automorphisms of $D$ is a "complete isometry algebra in standard position". This implies that $D$ carries a riemannian metric $\tilde{g}$ with nonpositive sectional curvature satisfying Lie $\operatorname{Iso}(D, \tilde{g})=$ Lie Aut $D$. We determine all Kähler metrics $f$ on $D$ for which the group $\operatorname{Aut}(D, f)$ of holomorphic isometries acts transitively. We prove that in this case $\operatorname{Aut}(D, f)$ contains a simply transitive split solvable subgroup.

The results of this paper are used to prove the fundamental conjecture for homogeneous Kähler manifolds admitting a solvable transitive group of automorphisms.
\end{abstract}

This paper is a continuation of [4]. We determine the Lie algebras of all simply transitive groups of automorphisms of a homogeneous Siegel domain $D$ and all Kähler metrics on $D$ for which the group of holomorphic isometries acts transitively.

This paper is organized as follows. In $\S 1$ we prove that the standard normal $j$-algebras of $[\mathbf{4}]$ are NC algebras [2] and that the Lie algebra of all automorphisms of $D$ is a complete isometry algebra in standard position in the sense of $[2]$. This implies that $D$ carries a riemannian metric $\tilde{g}$ with nonpositive sectional curvature satisfying Lie Iso $(D, \tilde{g})=$ Lie Aut $D$. In $\S 2$ we apply these results to prove that the Lie algebra of any simply transitive group of automorphisms of $D$ is a modification of a standard normal $j$-algebra. We also prove several technical results which are used in $[\mathbf{1 0}]$ to prove the fundamental conjecture of $[\mathbf{1 3}]$ for homogeneous Kähler manifolds admitting a solvable transitive group of automorphisms. In $\S 3$ we describe all homogeneous Kähler metrics on $D$. In particular we prove that each solvable Kähler algebra $(s,\{\cdot, \cdot\} j)$ generating a transitive group of automorphisms of a Siegel domain is a $j$-algebra and $\{x, y\}=w([j x, y])$. If $\{\cdot, \cdot\}$ is derived from the Bergman metric, this result has been proven by Koszul $[\mathbf{1 7}]$. Moreover, for any homogeneous Kähler metric $f$ on $D$ we get a simply transitive split solvable subgroup $S$ of $\operatorname{Aut}(D, f)$.

I would like to thank J. E. D'Atri for various helpful discussions and comments.

\section{NC algebras and complete isometry algebras.}

1.1. In this section we consider the normal $j$-algebra $\mathfrak{s}$ defined in $[4,12]$ and compare it to $\mathrm{NC}$ algebras in the sense of $[2,3.4]$. We would like to point out that the inner product $\langle\cdot, \cdot\rangle$ on $\mathfrak{s}$ is induced from the Bergman metric.

Received by the editors October 10, 1983.

1980 Mathematics Subject Classification. Primary 53B35, 32M99.

Key words and phrases. Homogeneous bounded domain, Kähler metric, solvable transitive group of automorphisms. 
LEMMA. $\mathfrak{s}$ is an $N C$ algebra without flat part. The inner product on $\mathfrak{s}$ associated with the Kähler structure is admissible for the NC algebra structure.

Proof. As usual we put $\mathfrak{n}=[\mathfrak{s}, \mathfrak{s}]$ and $\mathfrak{a}=\mathfrak{n}^{\perp}$. From $[\mathbf{4}, 14]$ we get that $\mathfrak{a}$ is abelian and acts selfadjointly on $\mathfrak{s}$ relative to the inner product $\langle\cdot, \cdot\rangle$ induced on $\mathfrak{s}$ from $[\mathbf{4}, 8]$. This shows (i) and (ii) of $[\mathbf{2}, 3.4]$. We choose $d_{1}, \ldots, d_{r}$ to be a complete set of primitive orthogonal idempotents as in $[\mathbf{4}, \mathbf{1}]$. We choose real numbers $\beta_{1}>\beta_{2}>\cdots>\beta_{r}$ and set $h_{0}=\sum_{i=1}^{r} \beta_{r} d_{r}$. Then $h_{0} \in \mathcal{L}$ and $X_{-1}\left[h_{0}\right] \in \mathfrak{s}_{-1}$; hence $H_{0}=j X_{-1}\left[h_{0}\right] \in \mathfrak{a}$ by $[\mathbf{4}, 13,14]$. From (15) and (16) of $[\mathbf{4}]$ we get $\alpha\left(H_{0}\right)>0$ for all roots $\alpha$. This proves (iii) of $[2,3.4]$. The condition (iv) of loc. cit. is void because $\mathfrak{a}$ has not the root 0 on $\mathfrak{n}$. To verify the last condition (v) of loc. cit. we note that $\mathfrak{n}_{\alpha}^{0}$ is clearly $\mathfrak{a}$-invariant. Therefore the orthogonal complement $\mathfrak{n}_{\alpha}^{1}$ of $\mathfrak{n}_{\alpha}^{0}$ in $\mathfrak{n}_{\alpha}$ is also $\mathfrak{a}$-invariant. This shows that $\mathfrak{s}$ is an NC algebra. Moreover, from the above it also follows immediately that $\langle\cdot, \cdot\rangle$ is admissible in the sense of $[\mathbf{2}, 3.7]$. Finally, from their definition it follows here that $\mathfrak{a}_{0}$ and $\mathfrak{n}_{0}$ of $[\mathbf{2}$, 4.3 vanish. This shows that $\mathfrak{s}$ has no flat part.

1.2. Next we consider a basic isometry algebra in the sense of $[\mathbf{2}, 5.2]$. The Lie algebras $\mathfrak{h}_{s s}$ and $\mathfrak{s}_{r}$ are defined in $[\mathbf{4}, 21]$.

LEMMA. $\mathfrak{h}_{s s}+\mathfrak{s}_{r}$ is a basic isometry algebra.

PROOF. We define $\mathfrak{s}_{s s}$ as in $[\mathbf{4}, 21]$ and have from $[\mathbf{4}, 31]$ that $\mathfrak{s}_{s s}$ is an Iwasawa subalgebra of $\mathfrak{h}_{s s}$. From the remark after $[\mathbf{4}, 21]$ we get that $\mathfrak{h}_{s s}$ is semisimple and has no compact ideal. This verifies $[\mathbf{2}, 5.2(\mathrm{i})]$. From $[\mathbf{4}, 23]$ we know $\mathfrak{s}=\mathfrak{s}_{s s}+\mathfrak{s}_{r}$ and the Lemma in $\S 1.1$ shows that $\mathfrak{s}$ is an $\mathrm{NC}$ algebra without flat part. This verifies $[\mathbf{2}$, 5.2(ii)]. It now suffices to show that $\mathfrak{h}_{s s}+\mathfrak{s}_{r}$ is a Lie algebra with radical $\mathfrak{s}_{r}$. We only have to prove $\left[\mathfrak{h}_{s s}, \mathfrak{s}_{r}\right] \subset \mathfrak{s}_{r}$. Now [8, I, Corollary 8.3] shows that it suffices to prove $\left[\mathfrak{h}_{s s}, L\left(\operatorname{center}\left(\chi \ominus \mathcal{P}_{1}\right)\right)\right]=0$. This follows from the fact that $L\left(\operatorname{center}\left(\chi \ominus \mathcal{P}_{1}\right)\right) \subset Z_{1}$ by $\left[\mathbf{8}, \mathrm{I}\right.$, Theorem 4.12] and $[\mathfrak{g}, \mathfrak{g}] \cap \operatorname{rad} \mathfrak{g} \subset \mathfrak{n}^{\phi}+\mathfrak{r}_{-1}+\mathfrak{r}_{-1 / 2} \subset \mathfrak{s}_{r}$ by standard results about Lie algebras $[\mathbf{3}, \S 5,3]$, where $\mathfrak{g}$ is defined at the beginning of $[\mathbf{4}, \S 1]$. This proves the assertion.

1.3. Eventually we will prove that $\mathfrak{g}$ is a complete isometry algebra in standard position. One of the conditions that has to be verified is

LEMMA. $\mathfrak{h}_{s s}+\mathfrak{s}_{r}$ is a nonexceptional basic isometry algebra.

PROOF. Assume the algebra would be exceptional. Then by $[2,7.6]$ we have $\left[\mathfrak{h}_{s s}, \mathfrak{s}_{r}\right]=0,\left[\mathfrak{s}_{r}, \mathfrak{s}_{r}\right]$ has codimension 1 in $\mathfrak{s}_{r}$ and there is $H_{0} \in \mathfrak{s}_{r}$ satisfying ad $H_{0} \mid\left[\mathfrak{s}_{r}, \mathfrak{s}_{r}\right]=$ Id. In particular we have $\left[\mathfrak{g}^{\prime \prime}, \mathfrak{s}_{r}\right]=0$, where $\mathfrak{g}^{\prime \prime}$ is defined in $[\mathbf{4}$, 17]. We know $e^{\prime} \in \mathcal{L}^{\prime}=\mathcal{P}_{1}$, where $X_{-1}\left[e^{\prime}\right] \in \mathfrak{g}_{-1}^{\prime \prime}$ and $j X_{-1}\left[e^{\prime}\right]=\left(T\left(e^{\prime}\right), \hat{T}\left(e^{\prime}\right)\right) \in$ $\mathfrak{g}_{0}^{\prime \prime}$. Hence $\left[j X_{-1}\left[e^{\prime}\right], \mathfrak{n}^{\phi}\right]=0$ implies $e^{\prime} \mathfrak{L}_{q j}=0$ for $j \neq 1$. This implies that the homogeneous Siegel domain $D$ is the product of the symmetric domain $D^{\prime}$ introduced before (17) of $[4]$ and a homogeneous Siegel domain $D^{*}$ having only affine infinitesimal automorphisms. Next we consider $\mathfrak{q}$ as defined after $[\mathbf{4}, 19]$. We know $\mathfrak{q} \subset \mathfrak{h}_{s s}$ by the definition of $\mathfrak{h}_{s s}$. Hence $\left[\mathfrak{q}, \mathfrak{s}_{r}\right]=0$. We know $\left(L(x), \frac{1}{2} \phi(x)\right) \in \mathfrak{q}$ for all $x \in \mathcal{L}_{11}+\cdots+\mathcal{L}_{q q}^{0}=\hat{\mathcal{L}}$ which are orthogonal to the center of $\hat{\mathcal{L}}$. This follows from [8, Theorem 4.12]. Our condition implies $x_{i i} \mathcal{L}_{i j}=0$ for all $i \neq j$ and $x_{i i}$ as above because $\left[\left(L\left(x_{i i}\right), \frac{1}{2} \phi\left(x_{i i}\right)\right), X_{-1}\left[y_{i j}\right]\right]=0$ and $X_{-1}\left[y_{i j}\right] \in \mathfrak{r}_{-1}$ by $[\mathbf{8}$, Theorem 8.1]. Let $D_{l}$ denote the simple summands of $\hat{\mathcal{L}}$. If $D_{l} \subset \mathcal{L}_{i i}$ and $D_{l} \mathcal{L}_{i j}=0$ for all $j \neq i$, then $D_{l}$ is a direct summand of $\mathcal{L}$ and, by the definition of $\mathcal{L}_{j j}$, would 
be contained in $\mathcal{L}_{q q}$. It would even be a part of $\mathcal{L}^{\prime}$. Hence such a $D$ does not exist in $\hat{\mathcal{L}}$. On the other hand $\left\{x_{i i} \in \mathcal{L}_{i i} ; x_{i i} \mathcal{L}_{i j}=0\right.$ for $\left.i \neq j\right\}$ is an ideal of $\mathcal{L}_{i i}$. This proves that this ideal is 0 . In particular, there does not exist an element in $\hat{\mathcal{L}}$ orthogonal to its center. Therefore $\hat{\mathcal{L}}$ is associative. Next we exploit the assumption that $\left[\mathfrak{s}_{r}, \mathfrak{s}_{r}\right]$ has codimension 1 in $\mathfrak{s}_{r}$. From the definition of $\mathfrak{s}_{r}$ in $[\mathbf{8}$, 21] we derive that the center of $\chi \ominus P_{1}$ has codimension 1. This implies $q=1$ and $\hat{\mathcal{L}}=\mathcal{L}_{q q}^{0} \cong \mathbf{R}$ or $q=2$ and $\hat{\mathcal{L}}=\mathcal{L}_{11} \cong \mathbf{R}$. The second case is impossible because we have shown above $e^{\prime} \mathcal{L}_{12}=0$. But in the first case we see that $D^{*}$ is quasi-symmetric because the corresponding algebra is $\mathcal{L}_{11}^{0} \cong \mathbf{R}$. Moreover, because this algebra is one dimensional, $D^{*}$ is even symmetric (note that $[\mathbf{8}, \mathrm{II}$, Theorem $3.3(3)]$ is obviously satisfied). Therefore $D$ is symmetric and $\mathfrak{s}_{r}=\mathfrak{s} \cap \operatorname{rad} \mathfrak{g}=0$. This is a contradiction and the lemma is proven.

1.4. In this section we show that the theory of bounded homogeneous domains fits into the larger frame of homogeneous riemannian manifolds with nonpositive curvature.

THEOREM. $\mathfrak{g}=\mathfrak{h}_{s s}+\mathfrak{t}_{a}+\mathfrak{s}_{r}$ is a complete isometry algebra in standard position.

PROOF. By $[\mathbf{2}, 7.6] \mathfrak{g}$ is a complete isometry algebra if $\mathfrak{g}$ has a stabilizer presentation for $\left(\mathfrak{h}_{s s}, \mathfrak{t}_{a}, \mathfrak{s}_{r},\langle\cdot, \cdot\rangle\right)$ with $\mathfrak{h}_{s s}+\mathfrak{s}_{r}$ a nonexceptional basic isometry algebra. The last condition was just verified. Hence it suffices to prove the stabilizier presentation property. We have to verify the conditions (i)-(iv) of $[\mathbf{2}, 7.4]$. We know that $\mathfrak{h}_{s s}+\mathfrak{s}_{r}$ is a basic isometry algebra. Hence it suffices to prove that $s_{r}$ is an ideal in $\mathfrak{g}$. We only have to show $\left[\mathfrak{t}_{a}, \mathfrak{s}_{r}\right] \subset \mathfrak{s}_{r}$. But this is clear because $\mathfrak{t}_{a}$ acts linearly on $\mathcal{L}$, leaves all $\mathcal{L}_{i j}$ invariant and annihilates $\mathcal{X}$. Hence (i). To verify (ii) we have to show that $\langle\cdot, \cdot\rangle$ on $\mathfrak{s}_{r}$ is $\left(\mathfrak{h}_{s s}, \Theta\right)$-admissible for some Cartan involution $\Theta$ of $\mathfrak{h}_{s s}$. But (19) and (24) of [4] define a Cartan involution $\Theta$ of $\mathfrak{h}_{s s}$. We have to verify the conditions of $[\mathbf{2}, 7.2]$. The first condition is satisfied because of $[\mathbf{4}$, $29]$ and the fact that $L\left(\operatorname{center}\left(\chi \ominus \mathcal{P}_{1}\right)\right)$ commutes with $\mathfrak{h}_{s s}=\mathfrak{g}^{\prime \prime}+\mathfrak{q}$. The second condition is just the lemma of $[\mathbf{4}, \S 1]$. The third condition is in our case equivalent to $\left[\mathfrak{h}_{s s}+\mathfrak{a}_{r}, \mathfrak{n}_{r}^{1}\right] \subset \mathfrak{n}_{r}^{1}$. But for $\mathfrak{a}_{r} \subset \mathfrak{a}$ this is clear and for $\mathfrak{h}_{s s}$ it follows from the fact $\left[\mathfrak{h}_{s s}, \mathfrak{n}_{r}\right] \subset \mathfrak{n}_{r}^{1}$ which has been mentioned in $[\mathbf{2}, 7.2]$.

To verify the last condition we only have to note that $\mathfrak{a}_{r}$ acts selfadjointly relative to $\langle\cdot, \cdot\rangle$ on $\mathfrak{s}_{r}$. hence $\langle\cdot, \cdot\rangle$ is $\left(\mathfrak{h}_{s s}, \Theta\right)$-admissible.

We continue by considering (iii) of $[2,7.4]$. We have to prove that $t_{a}$ equals the set $\mathfrak{u}$ of skew-adjoint derivations of $\mathfrak{s}_{r}$ which commute with ad $\mathfrak{h}_{s s} \mid \mathfrak{s}_{r}$. From the definition of $\boldsymbol{t}_{a}$ it is clear that it acts on $\mathfrak{s}_{r}$ by skew-adjoint derivations. Also from the definition of $\mathfrak{t}_{a}$ one easily derives that $\mathfrak{t}_{a}$ commutes with $\mathfrak{g}^{\prime \prime}$ and $q$. Hence $\mathfrak{t}_{a} \subset \mathfrak{u}$. Now let $X \in \mathfrak{u}$. We know that each element of $\mathfrak{a}$ is the sum of an element of $\mathfrak{h}_{s s}$ and an element of $\mathfrak{a}_{r}$. By assumption, $X$ commutes with ad $\mathfrak{h}_{s s} \mid \mathfrak{s}_{r}$. It is easy to see that $\mathfrak{s}_{r}$ is an NC algebra without flat part. Hence we can apply $[\mathbf{2}$, Lemma 5.5] and see that $X \mathfrak{a}_{r}=0$. Hence $X$ commutes with the action of $\mathfrak{a}_{r}$ on $\mathfrak{s}_{r}$. Altogether $X$ commutes with the action of $\mathfrak{a}$ on $\mathfrak{s}_{r}$. Therefore $X$ leaves invariant the root spaces of $\mathfrak{a}$ in $\mathfrak{s}_{r}$. In particular, $X$ leaves invariant each of the subspaces $\mathfrak{B}_{i j}$ contained in $\mathfrak{r}_{-1}$ and each of the subspaces $\phi\left(d_{j}\right) \mathcal{U}, d_{j} \notin \mathcal{P}_{1}$. We define an endomorphism $(Y, \hat{Y})$ on $\mathcal{L} \oplus \mathcal{U}$ via the action of $X$ on $\mathfrak{g}_{-1} \oplus \mathfrak{g}_{-1 / 2}$, where we extend $X$ from $\mathfrak{r}_{-1} \oplus \mathfrak{r}_{-1 / 2}$ by setting it equal to 0 on the orthogonal complement. It is easy to see that $(Y, \hat{Y})$ leaves $\mathcal{L}$ and $\mathcal{U}$ invariant, is skew-adjoint 
on $\mathcal{L} \oplus \mathcal{U}$ and annihilates $\mathcal{P}_{1} \oplus \mathcal{P}_{1 / 2}$. Moreover, from $\left[\operatorname{ad}\left(\mathfrak{h}_{s s}+\mathfrak{a}_{r}\right) \mid \mathfrak{s}_{r}, X\right]=0$ one gets $\left[(Y, \hat{Y}),\left(L(x), \frac{1}{2} \phi(x)\right)\right]=0$ for all $x \in X$. In particular, we have $0=[Y, L(X)]$ for all $x \in \chi$. Applying this to the identity $e \in \mathcal{X}$ we get $0=Y x-x Y e$ for all $x \in \mathcal{X}$. One knows that $\mathbf{R} X_{-1}\left[d_{l}\right]$ is a root space for $\mathfrak{a}$ in $\mathfrak{g}_{-1}$. Hence $Y d_{l} \in \mathbf{R} d_{l}$ for all $l$. But $Y$ is skew-adjoint, whence $Y d_{l}=0$. We know that $e$ is the sum of all $d_{l}$. Hence $Y e=0$ and $Y \mathcal{X}=0$ follows. Let $b \in \chi^{\perp}$; then $X_{-1}[b] \in \mathfrak{r}_{-1}$ and $j X_{-1}[b]=(T(b), \hat{T}(b)) \in \mathfrak{s}_{r}$. Hence we also have $X j X_{-1}[b] \in \mathfrak{s}_{r}$. Because $X$ leaves invariant $\mathfrak{g}_{0} \cap \mathfrak{s}_{r}$ and annihilates $\mathfrak{a}_{r}$ we get $X j X_{-1}[b]=j X_{-1}[f]$ with some $f \in \mathcal{L}$ such that $X_{-1}[f] \in \mathfrak{r}_{-1}$. Therefore

$$
\begin{aligned}
-X_{-1}[f] & =\left[j X_{-1}[f], X_{-1}[e]\right]=\left[X j X_{-1}[b], X_{-1}[e]\right] \\
& =X\left[j X_{-1}[b], X_{-1}[e]\right]-\left[j X_{-1}[b], X X_{-1}[e]\right] \\
& =-X X_{-1}[b]-\left[j X_{-1}[b], X_{-1}[Y e]\right]=-X X_{-1}[b] .
\end{aligned}
$$

This shows $X j z=j X z$ for all $z \in \mathfrak{r}_{-1}$. Next we want to prove $Y i u=i Y u$ for all $u \in \mathcal{U}$. It suffices to verify this for $u \in \mathcal{U}_{l}=\phi\left(d_{l}\right) \mathcal{U}$. We write $\hat{Y}=A+B$, where $A$ is $\mathbf{C}$-linear and $B$ is $\mathbf{C}$-antilinear. For $u, w \in \mathcal{U}_{l}$ we know $\left[X_{-1 / 2}[u], X_{-1 / 2}[w]\right]=$ $-4 X_{-1}[\operatorname{Im} F(u, w)]$, where $F(u, w) \in \mathbf{C} d_{l}$. Therefore $X$ annihilates this commutator. Evaluating the fact that $X$ is a derivative yields $\operatorname{Im} F(\hat{Y} u, w)+\operatorname{Im} F(u, \hat{Y} w)=$ 0 . Replacing $u$ by $i u$ gives $0=\operatorname{Re} F(A u, w)-\operatorname{Re} F(B u, w)+\operatorname{Re} F(u, \hat{Y} w)$. On the other hand, substituting $i w$ for $w$ we get $0=-\operatorname{Re} F(\hat{Y} u, w)-\operatorname{Re} F(u, A w)+$ $\operatorname{Re} F(u, B w)$. By a comparison of the last two equations we obtain $\operatorname{Re} F(B u, w)=$ $\operatorname{Re} F(u, B w)$. Multiplying an argument with $i$ yields $\operatorname{Im} F(B u, w)=-\operatorname{Im} F(u, B w)$. Hence $F(B u, w)=\overline{F(u, B w)}=F(B w, u)$. We use $\rho$ as defined in [4] preceding (1) and get $\rho(B u, w)=\sigma(F(B u, w), e)=\sigma(F(B w, u))=\rho(B w, u)$. We know that $\hat{Y}$ is skew-adjoint relative to $\rho$, therefore $B$ is skew-adjoint relative to $\rho$ and we get $\rho(B u, w)=-\rho(w, B u)=\overline{-\rho(B u, w)}$. Hence $\operatorname{Re} \rho(B u, w)=-\operatorname{Re} \rho(B u, w)$ whence $\rho(B u, w)=0$. This implies $B=0$ and $\hat{Y}$ is C-linear. A straightforward evaluation of $X\left[F_{-1 / 2}, G_{-1 / 2}\right]=\left[X F_{-1 / 2}, G_{-1 / 2}\right]+\left[F_{-1 / 2}, X G_{-1 / 2}\right]$ yields $Y F(u, w)=F(\hat{Y} u, w)+F(u, \hat{Y} w)$ for all $u, w \in \mathcal{U}$. To prove $(Y, \hat{Y}) \in \mathfrak{t}_{a}$ it now suffices to show that $Y$ is an infinitesimal automorphism of the cone $\Omega$ associated with $D$. Using the inductive construction of cones $[\mathbf{5}, \mathbf{6}, \mathbf{7}]$ we see that, because $Y \mathcal{X}=0$, it suffices to show that $Y$ is a derivation of each of the algebras $\mathcal{L}_{e r}^{(r)}$, where this is the mutation of the algebra $\mathcal{L}^{(r)}=\bigoplus_{1 \leq m, n \leq r} \mathcal{L}_{m n}$. We have only to consider expressions of type $L_{e_{r}}\left(x_{r n}\right) y_{r m}$, where $n, m<r$, because $Y$ commutes with $L(\mathcal{X})$. We note that $L_{e_{r}}\left(x_{r n}\right)$ is the $\mathcal{L}$-component of $j X_{-1}\left[x_{r n}\right]$. Hence

$$
\begin{aligned}
X_{-1}\left[Y L_{e_{r}}\left(x_{r n}\right) y_{r m}\right] & =X X_{-1}\left[L_{e_{r}}\left(x_{r n}\right) y_{r m}\right]=X\left[j X_{-1}\left[x_{r n}\right], X_{-1}\left[y_{r m}\right]\right] \\
& =\left[j X_{-1}\left[Y x_{r n}\right], X_{-1}\left[y_{r m}\right]\right]+\left[j X_{-1}\left[x_{r n}\right], X_{-1}\left[Y y_{r m}\right]\right],
\end{aligned}
$$

where we have used that all vector fields are contained in $\mathfrak{s}_{r}$ and that $X$ is a derivation of $\mathfrak{s}_{r}$ which commutes with $j$. Rewriting the right-hand side shows that $Y$ is a derivation of the algebras under consideration. This finishes the proof that $X$ is contained in $\mathfrak{t}_{a}$. Hence we have verified (iii) of $[\mathbf{2}, 7.4]$. The last remaining condition there is $\left[\mathfrak{h}_{s s}, \mathfrak{t}_{a}\right]=0$ and that the sum $\mathfrak{h}_{s s}+\mathfrak{t}_{a}+\mathfrak{s}_{r}$ is a direct sum of vector spaces.

Using the definitions of the summands both properties are easily verified. This finishes the proof that $\left(\mathfrak{h}_{s s}, \mathfrak{t}_{a}, \mathfrak{s}_{r},\langle\cdot, \cdot\rangle\right)$ is a stabilizer presentation of $\mathfrak{g}$. Finally, we 
have to prove that the complete isometry algebra $\mathfrak{g}$ is in standard position. But $\operatorname{trace}_{\mathfrak{s}_{r}}(D \operatorname{ad} a)=0$ for all skew-symmetric derivations $D$ of $\mathfrak{s}_{r}$ and $a \in \mathfrak{a}_{r}$ because $\operatorname{ad} a \mid \mathfrak{s}_{r}$ is selfadjoint.

1.5. In this section we check $\langle\cdot, \cdot\rangle$ for the various properties discussed in $[2,8.2$, pp. 74, 75].

We note that $\mathfrak{s}=\mathfrak{s}_{s s}+\mathfrak{s}_{r}$ and $\mathfrak{s}_{s s}$ and $\mathfrak{s}_{r}$ are orthogonal. Thus (i) is satisfied. We have shown in the proof of the theorem in $\S 1.4$ that $\langle\cdot, \cdot\rangle$ is $\left(\mathfrak{h}_{s s}, \Theta\right)$-admissible. Hence (iii) and (iii)' are satisfied. We can also verify (iv)'. Because ad $\mathfrak{a}_{r} \mid \mathfrak{s}_{r}$ is selfadjoint it suffices to prove that $N(H)$ as defined in $[2,8.2(8)]$ is positive semidefinite for all $H \in \mathfrak{a}_{r}$. We have $N(H)=\left(\operatorname{ad} H \mid \mathfrak{s}_{r}\right)^{2}$ whence $N(H) \geq 0$.

From this it also follows that $\sigma(H)$ and $N(H)$ are in general not positive definite. One can also check that the roots of $\mathfrak{a}_{r}$ in $\mathfrak{s}_{r}$ are not acute (only satisfy $\geq 0$ ). Hence (iv) is not satisfied. We also note that (ii) is not satisfied in general. However, splitting $\mathfrak{h}_{s s}$ into simple summands and decomposing $\mathfrak{s}_{s s}$ accordingly we get that $\langle\cdot, \cdot\rangle$ coincides - up to a positive factor-on each summand with $B_{1}\left(\frac{1}{2}(X-\Theta X), \frac{1}{2}(Y-\Theta Y)\right)$, where $B_{1}$ denotes the Killing form of $\mathfrak{h}_{s s}$. By a remark in $[2,8.2]$ the use of such an inner product induces "only trivial modifications" in the results of $[2, \S 8]$. So $[2$, Lemma 8.4$]$ is still true (see the remark at the end of $[4, \S 2]$ and the remark after the proof of Theorem 1 of $[4])$. It is unclear, however, how far Proposition 8.5 of [2] still holds.

This means that there is no simple constructive way to deform $\langle\cdot, \cdot\rangle$ to yield a metric with nonpositive sectional curvature. However, as shown in [1] and also in $[2, \S 9]$, there is some way to obtain such a metric.

2. Simply transitive groups on bounded homogeneous domains. We retain the notation of $\S 1$. In particular $D$ is a homogeneous Siegel domain (= h.S.d.) and $\mathfrak{s}$ a standard normal $j$-algebra. Let $\tilde{g}$ denote any riemannian metric on $D$; then $\operatorname{Iso}(D, \tilde{g})$ denotes the group of isometries of $D$ relative to $\tilde{g}$. For a group $G$, by $G^{0}$ we denote its connected component. By $\mathfrak{g}$ we denote, as in [4], the Lie algebra of all infinitesimal automorphisms of $D$.

2.1. The following result is fundamental for later proofs.

THEOREM 1. There exists a riemannian metric $\tilde{g}$ on $D$ which yields nonpositive sectional curvature and satisfies Lie $\operatorname{Iso}(D, \tilde{g})=\mathfrak{g}$.

PROOF. We know that $\left(\mathfrak{h}_{s s}, \mathfrak{t}_{a}, \mathfrak{s}_{r},\langle\cdot, \cdot\rangle\right)$ is a standard position stabilizer presentation of the complete isometry algebra $\mathfrak{g}$ by the results of $\S 1$. Now the proof of [2, Theorem 9.3] shows that one can change $\langle\cdot, \cdot\rangle$ on $\mathfrak{s}_{r}$ and extend it to $\mathfrak{s}_{s s}$ so that for the resulting riemannian metric $\tilde{g}$ on $D$ we have Lie $\operatorname{Iso}(D, \tilde{g}) \cong \mathfrak{g}$. Because we started with a Lie algebra of complete vector fields on $D$ we even have equality.

LEMMA 2. Let $D$ b a h.S.d. and $S$ a connected and transitive Lie group of automorphisms of $D$. Then $S$ contains a connected solvable and simply transitive subgroup $S^{\prime}$ which is closed in $S$ and the isotropy subgroup $K_{p}$ of $S$ is maximal compact in $S$ for any point $p \in D$. If $S$ is solvable, then $K_{p}$ is commutative.

Proof. We choose $\tilde{g}$ as in the theorem above. Then $S \subset \operatorname{Iso}(D, \tilde{g})$. Now the assertion follows from [1, Proposition 2.5].

A Kähler metric $f$ on a complex manifold $M$ is called homogeneous if the group $\operatorname{Aut}(M, f)$ of biholomorphic isometries acts transitively on $M$. 
LEMMA 3. Let $D$ be a h.S.d. and $f$ a homogeneous Kähler metric on D. Then there exists a connected, closed solvable subgroup $\tilde{S} \subset \operatorname{Aut}(D, f)$ which acts simply transitive on $D$.

Proof. We choose $\tilde{g}$ as in the theorem. Then $[\operatorname{Aut}(D, f)]^{0} \subset[\operatorname{Aut} D]^{0}=$ $[\operatorname{Iso}(D, \tilde{g})]^{0}$ and the assertion follows by Lemma 2 .

REMARK. Let $\mathfrak{s}$ be our standard normal $j$-algebra as above. By Theorem 1 $(D, \tilde{g})$ is a "solvmanifold with negative curvature." From $[\mathbf{2}, 6.4]$ we get the notion of "standard position" of $S=\exp \mathfrak{s}$. By $[2,6.12] S$ is in standard position iff $\operatorname{trac}\left(E \operatorname{ad}_{\mathfrak{s}} H\right)=0$ for all $H \in \mathfrak{a}$ and all skew-adjoint derivations $E$ of $\mathfrak{s}$. Because $\operatorname{ad}_{\mathfrak{s}} H$ is semisimple with real eigenvalues and commutes with $E$ this condition is satisfied for $S$. Hence $S$ is in standard position.

We would like to point out that in the proof of $[\mathbf{2}, 6.12]$ the sentence "From Corollary $2.13,(14)$ is equivalent to $(15) B(\mathfrak{m}, \mathfrak{a})=0$." seems to be inconclusive to us. However, it is not hard to fill this gap.

2.2. The following results are mostly consequences of the theorem in 1.4. They will be used in $[\mathbf{1 0}]$.

We will call a solvable Lie algebra $\mathfrak{s}$ together with an inner product $\{\cdot, \cdot\}$ and an orthogonal endomorphism $J$ a solvable Kähler algebra if

$$
\begin{gathered}
J^{2}=-\mathrm{Id}, \\
{[J x, J y]=J[J x, y]+J[x, J y]+[x, y], \quad x, y \in \tilde{\mathfrak{s}},} \\
\{[x, y], J z\}+\{[y, z], J x\}+\{[z, x], J y\}=0, \quad x, y, z \in \tilde{\mathfrak{s}} .
\end{gathered}
$$

These axioms are what remains of the axioms of $[\mathbf{1 4}$, part II, $\S 1]$ when the isotropy algebra vanishes (see also [14, part II, §4]).

For the notion of a normal $j$-algebra we refer to $[\mathbf{1 6}]$.

LEMMA 1. Let $(\tilde{\mathfrak{s}},\{\cdot, \cdot\}, J)$ be a solvable Kähler algebra which generates a simply transitive group of automorphisms of a h.S.d. Then $\tilde{\mathfrak{s}}$ is the modification in the sense of $[\mathbf{2}, 6.2]$ of a normal $j$-algebra.

Proof. We choose $\tilde{g}$ as in Theorem 1 in $\S 2.1$. and denote by $\tilde{S}$ the connected simply transitive group on $D$ generated by $\tilde{\mathfrak{s}}$. Then $\tilde{S} \subset G^{0}$, where $G=\operatorname{Iso}(D, \tilde{g})$. Moreover, $\tilde{S}$ is closed in $G^{0}$ : let $g_{n} \in \tilde{S}$ converge to $g \in G$. We fix a point $p \in D$ and find $h \in \tilde{S}$ so that $g(p)=h(p)$. Then $h^{-1} g_{n}$ converges to $h^{-1} g$ which fixes $p$. But this implies that $h^{-1} g_{n}(p)$ converges to $p$. Using the canonical isomorphism from $\tilde{S}$ to $D$ we get that $h^{-1} g_{n}$ converges to id $\in \tilde{S}$. Hence $g_{n}$ converges to $h$ and $h=g \in \tilde{S}$. Now we can apply [2, Theorem 6.7] and get a unique subgroup $\tilde{S}^{\prime}$ of $G^{0}$ in standard position so that $\tilde{S}$ is a modification of $\tilde{S}^{\prime}$ (in the sense of [2]). We use [2, Corollary 6.10] and see that $\tilde{S}^{\prime}$ is conjugate to $S$, the simply transitive Lie group on $D$ which is generated by our standard normal $j$-algebra, by an inner automorphism of $G^{0}$. Hence $\tilde{\mathfrak{s}}^{\prime}=\operatorname{Lie} \tilde{S}^{\prime}$ is a normal $j$-algebra and the assertion is proven.

We continue to use the notation of the proof above. We may assume $\tilde{S}^{\prime}=S$, whence $\tilde{\mathfrak{s}}^{\prime}=\mathfrak{s}$. Hence $\tilde{S}$ is a modification of $S$. By $[\mathbf{2}, 6.2]$ this is equivalent to $\tilde{\mathfrak{s}}=$ Lie $\tilde{S}=\{x+\psi(x) ; x \in \mathfrak{s}\}$, where $\psi: \mathfrak{s} \rightarrow\left(\right.$ norm $\left._{\mathfrak{g}} \mathfrak{s}\right) \cap \mathfrak{k}$ is a homomorphism of 
Lie algebras. Here norm $\mathfrak{g} \mathfrak{s}$ is the normalizer of $\mathfrak{s}$ in $\mathfrak{g}$ and $\mathfrak{k}$ is the Lie algebra of any maximal compact subgroup of $G$. We will assume that this group is the stabilizer of the point $i e$, where $e$ is the identity of $\mathcal{L}$ as in $\S 1$.

Under these assumptions we prove

LEMMA 2. $\operatorname{norm}_{\mathfrak{g}} \mathfrak{s} \cap \mathfrak{k}_{i e}=\mathfrak{t}_{a}+\left\{(T, \hat{T}) \in \mathfrak{h}_{s s} ; T d_{r}=0\right.$ for all $\left.r\right\}$.

ProOF. Let $X \in \mathfrak{w}=\operatorname{norm}_{\mathfrak{g}} \mathfrak{s} \cap \mathfrak{k}_{i e}$. We write $X=X_{-1}+X_{-1 / 2}+X_{0}+$ $X_{1 / 2}+X_{1}$ according to the splitting of $\mathfrak{g}$. We know that $\mathfrak{g}_{-1}$ and $\mathfrak{g}_{-1 / 2}$ are contained in $\mathfrak{s}$. Hence we can assume $X_{\lambda}=0$ for $\lambda=-1,-\frac{1}{2}$. On the other hand $\left(L(e), \frac{1}{2} \phi(e)\right) \in \mathfrak{s} \cap \mathfrak{g}_{0}$; now a straightforward computation shows $X_{1 / 2}=0$ and $X_{1}=0$. Hence we get $X \in \mathfrak{g}_{0}$. From $\mathfrak{s}=\mathfrak{s}_{s s}+\mathfrak{s}_{r}$ an easy computation shows $\mathfrak{t}_{a} \subset \mathfrak{w}$. Therefore we only have to consider $X=(T, \hat{T}) \in \mathfrak{h}_{s s} \cap \mathfrak{w}$. Because $X \in \mathfrak{k}_{i e}$ we know that $X$ is skew-adjoint as an endomorphism on $\mathfrak{L} \oplus \mathcal{U}$. Now it is easy to verify that $X$ is a skew-adjoint derivation of $\mathfrak{s}$. Because $\mathfrak{s}$ is, by the lemma in $\S 1.1$, an NC algebra without flat part we can apply [2, Lemma 5.5] and obtain that $X$ annihilates $\mathfrak{a}$. But this implies $T d_{r}=0$ for all $r$. This proves that $\mathfrak{w}$ is contained in the right-hand side of the assertion. The opposite inclusion follows by a straightforward computation. We reformulate the above results so that they can be applied in $[\mathbf{1 0}]$.

LEMMA 3. The map $D: \mathfrak{s} \rightarrow \operatorname{Der} \mathfrak{s}, x \mapsto \operatorname{ad} \psi(x) \mid \mathfrak{s}$ has the following properties:

$$
\begin{gathered}
D(x) \text { is skew-adjoint relative to }\langle\cdot, \cdot\rangle, \\
{[D(x), j]=0, \quad x \in \mathfrak{s},} \\
{[D(x), D(y)]=0, \quad x, y \in \mathfrak{s},} \\
D(x) \mathfrak{a}=0, \quad x \in \mathfrak{s} .
\end{gathered}
$$

PROOF. It is clear that $\operatorname{ad} \psi(x) \mid \mathfrak{s}$ is a derivation of $\mathfrak{s}$. In the proof of Lemma 2 we have mentioned that it acts skew-adjoint on $\mathfrak{s}$. From $[2,6.1]$ we know that $\psi$ is a homomorphism from $\mathfrak{s}$ into norm $_{\mathfrak{g}} \mathfrak{s} \cap \mathfrak{k}_{i e}$. Hence $\{\operatorname{ad} \psi(x) \mid \mathfrak{s} ; x \in \mathfrak{s}\}$ is a solvable Lie algebra. On the other hand this Lie algebra consists of skew-adjoint endomorphisms. This proves (2.6). Because $\psi$ is a homomorphism, (2.7) follows from (2.6). Finally, (2.8) was shown in the proof of Lemma 2. Hence it remains to prove (2.5). But $\psi(x)=(T, T) \in\left\{(R, \hat{R}) \in \mathfrak{g}_{0} ; R d_{r}=0\right.$ for all $\left.r\right\}$. We know that $\psi(x)$ commutes with $(0, i$ Id $) \in \mathfrak{g}_{0}$; this implies that $D(x)$ commutes with $j$ on $\mathfrak{s}_{-1 / 2}$. For $x_{n m} \in \mathfrak{L}_{n m}$ we have $j x_{n m}=\left(T\left(x_{n m}\right), \hat{T}\left(x_{n m}\right)\right)$ by $[\mathbf{4}, 13]$, where the right-hand side is defined in $[4,10]$. Now a straightforward calculation shows $\operatorname{ad} \psi(x) j x_{n m}=j T x_{n m}=j$ ad $\psi(x) x_{n m}$. This proves (2.5).

REMARKS. (1) Note that $(2.8)$ implies $D(x) \mathfrak{s} \subset[\mathfrak{s}, \mathfrak{s}]$ whence $D(D(x) y)=0$ for all $x, y \in \mathfrak{s}$.

(2) Lemmas 1 and 3 show that $(\tilde{\mathfrak{s}},\langle\cdot, \cdot\rangle, j)$ is a modification of $(\mathfrak{s},\langle\cdot, \cdot\rangle, j)$ in the sense of $[10,3.1]$.

(3) We will continue our study of solvable Kähler algebras in §3. First we determine the possible inner products $\{\cdot, \cdot\}$ on $\tilde{\mathfrak{s}}$. 


\section{Homogeneous Kähler metrics.}

3.1. Let $f$ be a homogeneous Kähler metric on a h.S.d. D. Then there exists a group $\tilde{S} \subset \operatorname{Aut}(D, f)$ which acts simply transitive on $D$ by Lemma 3 of $\S 2.1$. We choose a base point $i e \in D$ and induce the inner product $\{\cdot, \cdot\}$ on $\tilde{\mathfrak{s}}=$ Lie $\tilde{S}$ from $f$ via the canonical isomorphism. Analogously we define the operator $j$ on $\tilde{\mathfrak{s}}$. Thus we get a solvable Kähler algebra $(\tilde{\mathfrak{s}},\{\cdot, \cdot\}, j)$. The Lie product in $\tilde{s}$ is denoted by $(\cdot, \cdot)$. By Lemma 1 of $\S 2.2$ we know that $\tilde{\mathfrak{s}}$ is a modification of a normal $j$-algebra $\mathfrak{s}$. We will show below that the endomorphisms $D(x)$ of Lemma 3 of $\S 2.2$ are also skewadjoint relative to $\{\cdot, \cdot\}$. Let $\mathfrak{a}$ be the orthogonal complement of $[\mathfrak{s}, \mathfrak{s}]$ in $\mathfrak{s}$ relative to $\langle\cdot, \cdot\rangle$ (as usual). We consider the adjoint action of $\mathfrak{a}$ in the Lie algebra $\tilde{\mathfrak{s}}$. Because $D(x) \mathfrak{a}=0$ we see that $\mathfrak{a} \subset \tilde{\mathfrak{s}}$ leaves the weight spaces of $\mathfrak{a} \subset \mathfrak{s}$ in $\mathfrak{s}$ invariant. By $[\mathbf{4}$, $\S 1]$ we know that the weights in $\mathfrak{s}$ are $0, \varepsilon_{k}, \frac{1}{2} \varepsilon_{k}, \frac{1}{2}\left(\varepsilon_{k}-\varepsilon_{l}\right)$ and $\frac{1}{2}\left(\varepsilon_{k}+\varepsilon_{l}\right)$. Moreover, $j$ acts on the weights and maps $\varepsilon_{k}$ to $0, \frac{1}{2} \varepsilon_{k}$ to $\frac{1}{2} \varepsilon_{k}$ and $\frac{1}{2}\left(\varepsilon_{k}-\varepsilon_{l}\right)$ to $\frac{1}{2}\left(\varepsilon_{k}+\varepsilon_{l}\right)$. We put $\tilde{\mathfrak{s}}_{k l}=\tilde{\mathfrak{s}}_{\left(\varepsilon_{k}+\varepsilon_{l}\right) / 2}, \quad k<l, \tilde{\mathfrak{s}}_{k k}=\tilde{\mathfrak{s}}_{\varepsilon_{k} / 2}, j \tilde{\mathfrak{s}}_{k l}=\tilde{\mathfrak{s}}_{\left(\varepsilon_{k}-\varepsilon_{l}\right) / 2}, \tilde{\mathfrak{s}}_{0 k}=\tilde{\mathfrak{s}}_{\varepsilon_{k} / 2}$ and $\tilde{\mathfrak{s}}_{00}=\mathfrak{a}$. We consider the skew form $\tilde{\rho}(x, y)=\{x, j y\}$ on $\tilde{\mathfrak{s}}$. From [14, part III, Lemma 9] we know

$$
\frac{d}{d t} \rho\left({ }^{t \operatorname{ad} a} u, e^{t \operatorname{ad} a} v\right)=\rho\left(a, e^{t \operatorname{ad} a}(u, v)\right) \quad \text { for } a, u, v \in \tilde{\mathfrak{s}}
$$

Here ad denotes the left multiplication in $\tilde{\mathfrak{s}}$.

3.2. We retain the notation of $\S 3.1$ and prove the following important technical result.

THEOREM. The weight spaces of $\mathfrak{a}$ in $\tilde{\mathfrak{s}}$ are orthogonal relative to $\{\cdot, \cdot\}$ and all $D(x)$ are skew-adjoint relative to $\{\cdot, \cdot\}$.

ProOF. We note that $\tilde{\mathfrak{s}}_{k k}$ is one dimensional and for some generator $c_{k}$ we have $\left[j c_{k}, c_{k}\right]=c_{k}$ in $\mathfrak{s}$. But $D(x)$ is skew-adjoint relative to $\langle\cdot, \cdot\rangle$ and leaves $\tilde{\mathfrak{s}}_{\varepsilon_{k}}$ invariant. Hence $D(x) c_{k}=0$ for all $k$ and $x$.

Let $s=\sum_{k} c_{k}$ be the principal idempotent of $\mathfrak{s}$. Then ad $j s$ has eigenvalues with real part $0, \frac{1}{2}, 1$. We put $\tilde{\mathfrak{s}}=\tilde{\mathfrak{s}}_{0}+\tilde{\mathfrak{s}}_{1 / 2}+\tilde{\mathfrak{s}}_{1}$. Then $j \tilde{\mathfrak{s}}_{\lambda}=\tilde{\mathfrak{s}}_{1-\lambda}$ and we claim

$$
\tilde{\mathfrak{s}}_{\lambda} \text { and } \tilde{\mathfrak{s}}_{\mu} \text { are orthogonal relative to }\{\cdot, \cdot\} \text { if } \lambda \neq \mu \text {. }
$$

We use $(*)$ for $u \in \tilde{\mathfrak{s}}_{1}$ and $v \in \tilde{\mathfrak{s}}_{1 / 2}+\tilde{\mathfrak{s}}_{1}$. Then $(u, v)=[u, v]=0$ whence $\rho\left(e^{t \operatorname{ad} j s} u, e^{t \text { ad } j s} v\right)=\rho(u, v)$. However, the left-hand side grows in $t$ at least like $e^{t}$, therefore $\rho(u, v)=0$. This implies $\left\{\tilde{\mathfrak{s}}_{1}, j\left(\tilde{\mathfrak{s}}_{1 / 2}+\tilde{\mathfrak{s}}_{1}\right)\right\}=0$. From this (1) easily follows.

Because $\{\cdot, \cdot\}$ is invariant under $j$ we only have to consider the weight spaces contained in $\tilde{\mathfrak{s}}_{1 / 2}$ and in $\tilde{\mathfrak{s}}_{1}$.

$$
\tilde{\mathfrak{s}}_{k k} \text { is orthogonal to } \tilde{\mathfrak{s}}_{n m} \text { relative to }\{\cdot, \cdot\} \text { if } n \neq m \text { or } n=m \neq k \text {. }
$$

In case $k \neq n, k \neq m$ we have $\left(c_{k}, j x_{n m}\right)=-D\left(j x_{n m}\right) c_{k}=0$. Now the left-hand side of $(*)$ grows exponentially like $e^{t}$ if $a=j c_{k}, u=c_{k}, v=j x_{n m}$; arguing as above we see that $\tilde{\mathfrak{s}}_{k k}$ and $\tilde{\mathfrak{s}}_{n m}$ are orthogonal. Next we consider $k=n<m$. Here $\left(c_{k}, j x_{k m}\right)=\left[c_{k}, j x_{k m}\right]=0$, because $\varepsilon_{k}+\frac{1}{2}\left(\varepsilon_{k}-\varepsilon_{m}\right)$ is not a weight. The same argument as above shows that $\tilde{\mathfrak{s}}_{k k}$ is orthogonal to $\tilde{\mathfrak{s}}_{k m}$ relative to $\{\cdot, \cdot\}$ if $k<m$. The last remaining case is $n<m=k$. 
Here we have

$$
\begin{aligned}
\rho\left(c_{k}, j x_{n k}\right) & =\rho\left(\left(j c_{k}, c_{k}\right), j x_{n k}\right)=-\rho\left(\left(c_{k}, j x_{n k}\right), j c_{k}\right)-\rho\left(\left(j x_{n k}, j c_{k}\right), c_{k}\right) \\
& =-\rho\left(\left[c_{k}, j x_{n k}\right], j c_{k}\right)-\rho\left(j\left(j x_{n k}, c_{k}\right)+j\left(x_{n k}, j c_{k}\right), c_{k}\right) \\
& =2 \rho\left(\left[j x_{n k}, c_{k}\right], j c_{k}\right)-\rho\left(\left(j c_{k}, x_{n k}\right), j c_{k}\right) \\
& =2 \rho\left(x_{n k}, j c_{k}\right)-\frac{1}{2} \rho\left(x_{n k}, j c_{k}\right)-\rho\left(D\left(j c_{k}\right) x_{n k}, j c_{k}\right) .
\end{aligned}
$$

Therefore $\frac{1}{2} \rho\left(x_{n k}, j c_{k}\right)=\rho\left(D\left(j c_{k}\right) x_{n k}, j c_{k}\right)$. Hence $\rho\left(\exp \left(t D\left(j c_{k}\right)\right) x_{n k}, j c_{k}\right)=$ $e^{t / 2} \rho\left(x_{n k}, j c_{k}\right)$. But the left-hand side is bounded whence $\rho\left(x_{n k}, j c_{k}\right)=0$. This finishes the proof of (2).

$$
\tilde{\mathfrak{s}}_{0 k} \text { and } \tilde{\mathfrak{s}}_{0 m} \text { are orthogonal relative to }\{\cdot, \cdot\} \text { if } k \neq m .
$$

We know $\left(u_{0 k}, v_{0 m}\right)=\left[u_{0 k}, v_{0 m}\right] \in \tilde{\mathfrak{s}}_{k m}$. Hence $\rho\left(j s e^{t \text { ad } j s}\left(u_{0 k}, v_{0 m}\right)\right)=0$ by (2). From $(*)$ we get $\rho\left(e^{t \text { ad } j s} u_{0 k}, e^{t \text { ad } j s} v_{0 m}\right)=\rho\left(u_{0 k}, v_{0 m}\right)$. But the left-hand side of this equation grows like $e^{t}$, whence the assertion.

$$
\tilde{\mathfrak{s}}_{k l} \text { and } \tilde{\mathfrak{s}}_{m n} \text { are pairwise orthogonal relative to }\{\cdot, \cdot\} \text {. }
$$

By (2) we can assume $k<l$ and $m \neq n$. But then it is easy to check that $\left(j x_{k l}, y_{m n}\right)=\left[j x_{k l}, y_{m n}\right]$ does not vanish only if $l=m$ or $l=n$. In this case the product lies in $\tilde{\mathfrak{s}}_{k n}\left(\right.$ resp. $\left.\tilde{\mathfrak{s}}_{k m}\right)$ and because we consider different weight spaces we have $k \neq n$ (resp. $k \neq m$ ). Therefore $\rho\left(j c_{k}, e^{t \text { ad } j s}\left(j \tilde{\mathfrak{s}}_{k l}, \tilde{\mathfrak{s}}_{m n}\right)\right)=0$ and from (*) we get

$$
\rho\left(e^{t \text { ad } j c_{k}} j x_{k l}, e^{t \text { ad } j c_{k}} y_{m n}\right)=\rho\left(j x_{k l}, y_{m n}\right) .
$$

We know that the real part of $\operatorname{ad} j c_{k}$ on $\tilde{\mathfrak{s}}_{m n}$ is 0 or $\frac{1}{2}$. Also

$$
\left(j c_{k}, j x_{k l}\right)=j\left(j c_{k}, x_{k l}\right)+j\left(c_{k}, j x_{k l}\right)=\frac{1}{2} j x_{k l}+j D\left(j c_{k}\right) x_{k l}
$$

because $\left(c_{k}, j x_{k l}\right)=\left[c_{k}, j x_{k l}\right]=0$. Therefore the left-hand side of the equation above grows at least like $e^{t / 2}$. As a consquence $\rho\left(j \tilde{\mathfrak{s}}_{k l}, \tilde{\mathfrak{s}}_{m n}\right)=0$ and we have verified that the weight spaces relative to $\mathfrak{a}$ in $\mathfrak{s}$ are pairwise orthogonal relative to $\{\cdot, \cdot\}$.

Now we want to prove that all $D(x)$ are skew-adjoint relative to $\{\cdot, \cdot\}$. We note that, by Lemma 3 in $\S 2.2, D(x)$ commutes with $j$. It therefore suffices to consider $D(x)$ on $\tilde{\mathfrak{s}}_{n m}$ and on $\tilde{\mathfrak{s}}_{0 k}$. Here we may assume $n<m$ as mentioned above. For $u_{n m}, v_{n m} \in \tilde{\mathfrak{s}}_{n m}$ we have $\left(j u_{n m}, v_{n m}\right) \in \tilde{\mathfrak{s}}_{n m}$. Hence ad $j c_{k}\left(j u_{n m}, v_{n m}\right)=$ $\delta_{k n}\left(j u_{n m}, v_{n m}\right)$. Therefore from $(*)$ we get

$$
\frac{d}{d t} \rho\left(e^{t \operatorname{ad} j c_{n}} j u_{n m}, e^{t \operatorname{ad} j c_{n}} v_{n m}\right)=e^{t} \rho\left(j u_{n m}, v_{n m}\right)
$$

An integration yields $\rho\left(e^{t \text { ad } j c_{n}} j u_{n m}, e^{t \text { ad } j c_{n}} v_{n m}\right)=e^{t} \rho\left(j u_{n m}, v_{n m}\right)+b$, where $b$ is a constant. Above we have shown $\left(j c_{n}, j u_{n m}\right)=\frac{1}{2} u_{n m}+j D\left(j c_{n}\right) u_{n m}$; we also know $\left(j c_{n}, v_{n m}\right)=\frac{1}{2} v_{n m}+D\left(j c_{n}\right) v_{n m}$. Hence

$$
e^{t} \rho\left(e^{t D\left(j c_{n}\right)} j u_{n m}, e^{t D\left(j c_{n}\right)} v_{n m}\right)=e^{t} \rho\left(j u_{n m}, v_{n m}\right)+b .
$$

This implies $b=0$. We note again that $D\left(j c_{n}\right)$ commutes with $j$. We obtain that $e^{t D\left(j c_{n}\right)}$ is orthogonal on $\tilde{\mathfrak{s}}_{n m}$. Assume now $k \neq n$. Then $\rho\left(j c_{k} e^{t \text { ad } j c_{k}}\left(j u_{n m}, v_{n m}\right)\right)$ $=0$ and $(*)$ yields $\rho\left(e^{t \text { ad } j c_{k}} j u_{n m}, e^{t \text { ad } j c_{k}} v_{n m}\right)=\rho\left(j u_{n m}, v_{n m}\right)$ after integration. We know

$$
\left(j c_{k}, v_{n m}\right)=\frac{1}{2} \delta_{k m} v_{n m}+D\left(j c_{k}\right) v_{n m}
$$


and

$$
\begin{aligned}
\left(j c_{k}, j u_{n m}\right) & =j\left(j c_{k}, u_{n m}\right)+j\left(c_{k}, j u_{n m}\right)=\frac{1}{2} \delta_{m k} j u_{n m}+j D\left(j c_{k}\right) u_{n m}-\delta_{m k} j u_{n m} \\
& =-\frac{1}{2} \delta_{m k} j u_{n m}+j D\left(j c_{k}\right) u_{n m} .
\end{aligned}
$$

As above we obtain that $e^{t D\left(j c_{k}\right)}$ is orthogonal.

3.3. We keep the notation of the previous sections.

THEOREM 1. There exists a linear form $\omega$ on $\tilde{\mathfrak{s}}$ so that

$$
\{x, y\}=\omega((j x, y)) \quad \text { for all } x, y \in \tilde{\mathfrak{s}} .
$$

ProOF. From the theorem in $\S 3.2$ we derive $D(x) \in \operatorname{Lie} \operatorname{Aut}(D, f)$. Hence $\mathfrak{s} \subset$ Lie $\operatorname{Aut}(D, f)$. Clearly, $(\mathfrak{s},\{\cdot, \cdot\}, j)$ is a normal Kähler algebra in the sense of $[\mathbf{1 4}$, part II, §4]. Moreover, because $(\mathfrak{s},\langle\cdot, \cdot\rangle, j)$ is a normal $j$-algebra we have the principal idempotent $s \in \mathfrak{s}$ which satisfies (1), (2) and (3) of the Fundamental Theorem of $[\mathbf{1 4}$, part III, §1]. We obtain now the assertion from [14, part III, Lemma 1]. We rephrase our results in

THEOREM 2. Let $f$ be a homogeneous Kähler metric on a h.S.d. D.

(a) There exists a simply transitive solvable subgroup $\tilde{S} \subset \operatorname{Aut}(D, f)$.

(b) Let $\tilde{S} \subset \operatorname{Aut}(D, f)$ be any simply transitive solvable Lie group on $D$ and $(\tilde{\mathfrak{s}},\{\cdot, \cdot\}, j)$ the corresponding Kähler algebra, where $\tilde{\mathfrak{s}}=$ Lie $S$ and $\{\cdot, \cdot\}$ is induced from $f$. Then $(\tilde{\mathfrak{s}},\{\cdot, \cdot\}, j)$ is a modification of a normal $j$-algebra $(\mathfrak{s},\{\cdot, \cdot\}, j)$, where $S=\exp \mathfrak{s} \subset \operatorname{Aut}(D, f)$.

(c) There exists a simply transitive split solvable subgroup $S \subset \operatorname{Aut}(D, f)$.

ProOF. (a) See Lemma 2 in $\S 2.1$,

(b) see Lemma 1 in $\S 2.2$ and Theorem 1 above,

(c) see (b).

3.4. We want to determine all possible $\omega$ 's. Here we consider the normal $j$ algebra $\mathfrak{s}$ with inner product $\{x, y\}=\omega([j x, y])$. We put $\mathfrak{a}=\mathfrak{s} \ominus[\mathfrak{s}, \mathfrak{s}]$ as usual. If $x \in \mathfrak{s}$ is orthogonal to $\mathfrak{a}+j a$, then there exists $a \in \mathfrak{a}$ so that $[a, x]=\lambda x, \lambda \neq 0$. This implies $\omega(x)=0$ if $\{x, \mathfrak{a}+j \mathfrak{a}\}=0$. The values of $\omega$ on $\mathfrak{a}$ are undetermined and on $j \mathfrak{a}$ we have $\left\{d_{k}, d_{k}\right\}=\omega\left(d_{k}\right)$, where $d_{k} \in j \mathfrak{a}$ satisfies $\left[j d_{k}, d_{k}\right]=d_{k}$. (Here $d_{k}$ is as in $[\mathbf{4}, \S 1]$.) Because $\omega$ is never evaluated on $\mathfrak{a}$ we may assume $\omega(\mathfrak{a})=0$. Hence $\omega$ is determined by $\tau=\left(-\tau_{1}, \ldots,-\tau_{r}\right)$, where $\tau_{m}=\omega\left(d_{m}\right)$.

Next we start with any tuple $\tau_{1}, \ldots, \tau_{r}$ of negative real numbers and consider the positive "compound power function" $\eta(x)=x^{\tau}=\prod_{k=1}^{r} \chi_{k}(x)^{\tau_{k}}$ in the sense of $[12, \S 2]$ on the cone $\Omega$ which is associated with the h.S.d. $D=D(\Omega, F)$. We know that $\eta$ is a homogeneous function and considering $\Omega$ as a subset of $\mathcal{L}$ we have $\eta\left(e^{t \operatorname{ad} j d_{k}} x\right)=e^{-t \tau_{k}} \eta(x)$ and $\eta\left({ }^{\operatorname{ad} u} x\right)=\eta(x)$ for all $u \in[\mathfrak{s}, \mathfrak{s}]$.

We put

$$
\eta_{x}^{\prime}(u)=-d_{x} \log \eta(u)=-\left.\frac{d}{d t} \log \eta(x+t u)\right|_{t=0}
$$

and $\eta_{x}^{\prime \prime}(u, v)=d_{x}^{2} \log \eta(u, v)$.

From [12, Proposition 2.3] we obtain that a certain power $\eta(x)^{\beta}$ of $\eta$ can be represented as

$$
\eta(x)^{\beta}=\gamma \int_{\Omega^{*}} e^{-\{x, y\}} \eta_{*}(y)^{\beta} d \mu(y)
$$


where $\gamma>0$ and $\Omega_{*}$ denotes the dual cone for $\Omega$. From this it follows that $\eta^{\beta}$ is "matrix positive" in the sense of $[\mathbf{1 1}, \S 5]$. In particular, $\eta^{\beta}$ can be extended holomorphically to $\Omega+i \mathcal{L}$ and $\eta_{x}^{\prime \prime}$ is a positive definite bilinear form on $\mathcal{L}$ (this last statement can be shown directly; then $[\mathbf{1 1}$, Theorem 9.2$]$ applies and $\eta^{\beta}$ is matrix positive for some $\beta>0)$.

We set $\psi\left(z_{1}, z_{2} ; w_{1}, w_{2}\right)=\frac{1}{2} i\left(z_{1}-\bar{w}_{1}\right)-F\left(z_{2}, w_{2}\right)$ for $\left(z_{1}, z_{2}\right),\left(w_{1}, w_{2}\right) \in D$ and consider the function $Q(z ; w)=\eta(\psi(z ; w))^{\beta}$. We put

$$
\tilde{f}_{w}(u, v)=\left.\frac{d}{d t} \frac{d}{d s} \beta^{-1} \operatorname{Re} \log Q(w+t u, w+s v)\right|_{t=s=0} .
$$

We note that we use here

$$
\frac{d}{d t} \log Q(w+t u, z)=Q(w+t u, z)^{-1} \frac{d}{d t} Q(w+t u, z) .
$$

A direct computation shows

$$
\begin{aligned}
\tilde{f}_{w}(u, v)=\operatorname{Re}\left(\eta_{\psi(w, w)}^{\prime \prime}\left(\left(\frac{1}{2} i\right) u_{1}-F\left(u_{2}, w_{2}\right), \overline{\left(\frac{1}{2} i\right) v_{1}-F\left(v_{2}, w_{2}\right)}\right)\right. & \\
- & \left.\eta_{\psi(w, w)}^{\prime}\left(F\left(u_{2}, v_{2}\right)\right)\right)
\end{aligned}
$$

In particular for the point $w=i e \in D$ we have $\psi(i e, i e)=e$ and

$$
\tilde{f}_{i e}(u, v)=\operatorname{Re}\left(\frac{1}{4} \eta_{e}^{\prime \prime}\left(u_{1}, \bar{v}_{1}\right)-\eta_{e}^{\prime}\left(F\left(u_{2}, v_{2}\right)\right)\right) \text {. }
$$

We note that from the above we know that $\tilde{f}$ is a Kähler metric on $D$. Using the explicit expressions for the transformations on $D$ corresponding to elements of $\mathfrak{s}_{0}, \mathfrak{s}_{1}, \mathfrak{s}_{1 / 2}$, where $\mathfrak{s}_{\lambda}=\mathfrak{s} \cap \mathfrak{g}_{-\lambda}$, we see $\psi\left(\mathcal{U}_{0} z ; \mathcal{U}_{0} w\right)=\mathcal{U}_{0} \psi(z ; w)$ for $\mathcal{U}_{0} \in \exp \mathfrak{s}_{0}$ and $\psi(\mathcal{U} z ; \mathcal{U} w)=\psi(z ; w)$ for $\mathcal{U} \in \exp \left(\mathfrak{s}_{1 / 2}+\mathfrak{s}_{1}\right)$. Hence $Q(\mathcal{U} z ; \mathcal{U} w)=\psi(\mathcal{U}) Q(z ; w)$ with some positive homomorphism $\alpha: S=\exp \mathfrak{s} \rightarrow \mathbf{R}^{+}$. From this it follows that $S$ consists of isometries relative to $\tilde{f}$.

We consider the inner product induced from $\tilde{f}$ on $s$ relative to $i e$

$$
\begin{aligned}
\{x, y\}_{0} & =\tilde{f}_{i e}(x(i e), y(i e)) \\
& =\operatorname{Re}\left(\frac{1}{4} \eta_{e}^{\prime \prime}\left(x(i e)_{1}, y(i e)_{1}\right)-\eta_{e}^{\prime}\left(F\left(x(i e)_{2}, y(i e)_{2}\right)\right)\right) .
\end{aligned}
$$

It is easy to see that $j$ is orthogonal relative to $\{\cdot, \cdot\}_{0}$.

We define $\omega_{0}(x)=-\frac{1}{4} \operatorname{Re} \eta_{e}^{\prime}(x(i e))$. We claim

$$
\{x, y\}_{0}=\omega_{0}([j x, y])
$$

First we note that the elements of $\mathfrak{s}_{1}$ map to $\mathcal{L}$, the elements of $\mathfrak{s}_{1 / 2}$ map to $\mathcal{U}$ and those of $\mathfrak{s}_{0}$ map to $i \mathcal{L}$. Hence from (3.3) it is clear that the spaces $\mathfrak{s}_{\lambda}$ are pairwise orthogonal relative to $\{\cdot, \cdot\}_{0}$. We use the explicit formulas for the vector fields corresponding to the elements of $\mathfrak{s}$ and compute their Lie brackets. Then we get

$$
\begin{gathered}
{\left[j X_{-1 / 2}\left[d_{1 / 2}\right], X_{-1 / 2}\left[w_{1 / 2}\right]\right]^{+}=4 X_{-1}\left[\operatorname{Im} F\left(i d_{1 / 2}, w_{1 / 2}\right)\right]^{+}} \\
{\left[j X_{-1}\left[a_{1}\right], X_{-1}\left[b_{1}\right]\right]^{+}=X_{-1}\left[T\left(a_{1}\right) b_{1}\right] .}
\end{gathered}
$$

Therefore

$$
\omega_{0}\left(\left[j d_{1 / 2}, w_{1 / 2}\right]\right)=-\eta_{e}^{\prime}\left(\operatorname{Re} F\left(d_{1 / 2}, w_{1 / 2}\right)\right)=\left\{d_{1 / 2}, w_{1 / 2}\right\}_{0}
$$

and

$$
\omega_{0}\left(\left[j a_{1}, b_{1}\right]\right)=-\frac{1}{4} \eta_{e}^{\prime}\left(T\left(a_{1}\right) b_{1}\right)
$$


From the transformation property of $\eta$ relative to $S$ we get

$$
-\eta_{x}^{\prime}(u)=\left.\frac{d}{d t} \log \eta(x+t u)\right|_{t=0}=\left.\frac{d}{d t} \log \eta\left(\mathcal{U}_{0} x+t \mathcal{U}_{0} u\right)\right|_{t=0}=-\eta_{\mathcal{U}_{0} x}^{\prime}\left(\mathcal{U}_{0} u\right) .
$$

We substitute $U_{0}=\exp t T$, differentiate for $t$ at $t=0$ and obtain $\eta_{x}^{\prime}(T u)=$ $-\eta_{x}^{\prime \prime}(T e, u)$. Hence $\omega_{0}\left(\left[j a_{1}, b_{1}\right]\right)=\frac{1}{4} \eta_{e}^{\prime \prime}\left(a_{1}, b_{1}\right)=\left\{a_{1}, b_{1}\right\}_{0}$. Finally $\omega_{0}\left(\left[j a_{0}, b_{0}\right]\right)=$ $\omega_{0}\left(\left[j j b_{0}, j a_{0}\right]\right)=\left\{j b_{0}, j a_{0}\right\}_{0}=\left\{a_{0}, b_{0}\right\}_{0}$.

$$
\omega_{0}\left(d_{k}\right)=\frac{1}{4} \tau_{k}
$$

We compute

$$
-\eta_{e}^{\prime}\left(d_{k}\right)=\left.\frac{d}{d t} \log \eta\left(\exp t T\left(d_{k}\right) e\right)\right|_{t=0}=\left.\frac{d}{d t} \log \left(e^{-t \tau_{k}} \eta(e)\right)\right|_{t=0}=-\tau_{k} .
$$

Hence the assertion.

Summing up we get

THEOREM 1. There is a canonical bijection between the set of all homogeneous Kähler metrics $f$ on $D$ with $\exp \mathfrak{s} \subset \operatorname{Aut}(D, f)$ and $\left(\mathbf{R}^{+}\right)^{r}$. Each homogeneous Kähler metric on $D$ can be derived from a function $\eta$ on $\Omega$.

REMARK. It would be interesting to know which of the homogeneous Kähler metrics on $D$ have nonpositive sectional curvature. We hope to pursue this topic in a separate publication.

Finally, comparing the above results with the definition $[15, \S 1]$ we have

THEOREM 2. Each solvable Kähler algebra on a h.S.d. is a simple j-algebra.

3.5. Let $(\tilde{\mathfrak{s}},\{\cdot, \cdot\}, J)$ be a solvable Kähler algebra generating a (simply) transitive group of automorphisms on a h.S.d. The Lie product in $\tilde{\mathfrak{s}}$ is denoted by $(\cdot, \cdot)$. We know from Lemma 1 in $\S 2.2$ that on the vector space $\tilde{\mathfrak{s}}$ we can define a normal $j$ algebra (with Lie product $[\cdot, \cdot]$ ) so that $(x, y)=[x, y]+D(x) y-D(y) x$ holds, where $D$ is a map having the properties stated in Lemma 3 in $\S 2.1$. We may assume that the normal $j$-algebra is our standard normal $j$-algebra $\mathfrak{s}$. We note however, that the inner product here is $\{\cdot, \cdot\}$. From the properties of $J$ on $\tilde{\mathfrak{s}}$ and $j$ on $\mathfrak{s}$ we see that $J=j$ holds.

LemmA. Let $\tilde{\mathfrak{s}}, \tilde{\mathfrak{s}},\{\cdot, \cdot\}$ and $j$ be as above. Then we have

(1) Each skew-adjoint derivation of $\tilde{\mathfrak{s}}$ which commutes with $j$ is also a skewadjoint derivation for $\mathfrak{s}$. In particular we have for such a derivation $D: D \tilde{\mathfrak{s}} \subset(\tilde{\mathfrak{s}}, \tilde{\mathfrak{s}})$ and $D \mathfrak{a}=D(\tilde{\mathfrak{s}} \ominus(\tilde{\mathfrak{s}}, \tilde{\mathfrak{s}}))=0$.

(2) Let $x \in \tilde{\mathfrak{s}}$ satisfy $(j x, x)=0$. Then $x=0$.

ProOF. (1) We know that $\mathfrak{s}$ and $\tilde{\mathfrak{s}}$ have the same weight spaces relative to $\mathfrak{a}$. Hence $D j \mathfrak{a}=0$ and also $D \mathfrak{a}=0$. Therefore $D$ commutes with the adjoint action of $\mathfrak{a}$ in $\tilde{\mathfrak{s}}$. This implies that $D$ commutes with the selfadjoint and the skew-adjoint part of $a d \mathfrak{a}$ in $\tilde{\mathfrak{s}}$. This implies the assertion because $\mathfrak{s}$ and $\tilde{\mathfrak{s}}$ have the same Lie products in $(\tilde{\mathfrak{s}}, \tilde{\mathfrak{s}})=[\mathfrak{s}, \mathfrak{s}]$.

(2) We choose $\omega$ as in Theorem 1 in $\S 3.3$. Then for any skew-adjoint derivation $D$ of $\mathfrak{s}$ we get $0=\{D s, y\}+\{s, D y\}=\{s, D y\}=\omega((j s, D y))=\omega(D y)$, where $s$ is the principal idempotent of $\mathfrak{s}$ and where we have used that $D \tilde{\mathfrak{s}} \subset(\tilde{\mathfrak{s}}, \tilde{\mathfrak{s}})$ and $\omega\left(\tilde{\mathfrak{s}}_{1 / 2}\right)=0$ holds. Hence $(j x, x)=0$ implies $0=\omega((j x, x))=\{x, x\}$. Therefore $x=0$. 


\section{BIBLIOGRAPHY}

1. R. Azencott and E. N. Wilson, Homogeneous manifolds with negative curvature. I, Trans. Amer. Math. Soc. 215 (1976), 323-362.

2. __ Homogeneous manifolds with negative curvature. II, Mem. Amer. Math. Soc. No. 178 (1976).

3. N. Bourbaki, Groupes et algèbres de Lie, Chapitre I, Hermann, Paris, 1971.

4. J. E. D'Atri and J. Dorfmeister, The isotropy representation for homogeneous Siegel domains, Pacific J. Math. (to appear).

5. J. Dorfmeister, Zur Konstruktion Homogener Kegel, Math. Ann. 216 (1975), 79-96.

6. $\ldots$, Inductive construction of homogeneous cones, Trans. Amer. Math. Soc. 252 (1979), 321 349.

7. __ Algebraic description of homogeneous cones, Trans. Amer. Math. Soc. 255 (1979), 61-89.

8. __ Homogene Siegel-Gebiete, Habilitationsschrift, Münster, 1978.

9. __ Homogeneous Siegel domains, Nagoya Math. J. 86 (1982), 39-83.

10. __ Homogeneous Kähler manifolds admitting a transitive solvable group of automorphisms, Ann. Sci. École Norm. Sup. (to appear).

11. J. Dorfmeister and M. Koecher, Reguläre Kegel, Jber. Deutsch. Math.-Verein. 81 (1979), 109151.

12. S. G. Gindikin, Analysis in homogeneous domains, Russian Math. Surveys 19 (1964), 1-89.

13. S. G. Gindikin and E. B. Vinberg, Kählerian manifolds admitting a transitive solvable automorphism group, Math. Sb. 74 (1967), 333-351.

14. S. G. Gindikin, I. I. Piatetskii-Shapiro and E. B. Vinberg, Homogeneous Kähler manifolds, C.I.M.E. Edizione Cremonese, Roma, 1967.

15. I. I. Piatetskii-Shapiro, Bounded homogeneous domains in n-dimensional complex space, Amer. Math. Soc. Transl. 43 (1964), 299-320.

16. __ Automorphic functions and the geometry of classical domains, Gordon and Breach, New York and London, 1969.

17. J. L. Koszul, Sur la forme hermitienne canonique des espaces homogènes complexes, Canad. J. Math. 7 (1955), 562-576. 